\title{
Cardiopatias Congénitas Complexas: Influência do Diagnóstico Pré-Natal
}

\author{
Complex Congenital Heart Disease: The Influence of Prenatal Diagnosis
}

\author{
Marta CORREIA ${ }^{1}$, Fabiana FORTUNATO², Duarte MARTINS ${ }^{3}$, Ana TEIXEIRA ${ }^{3}$, Graça NOGUEIRA ${ }^{3}$, Isabel MENEZES ${ }^{3}$, \\ Rui ANJOS ${ }^{3}$ \\ Acta Med Port 2015 Mar-Apr;28(2):158-163
}

\section{RESUMO}

Introdução: As cardiopatias congénitas complexas são patologias graves, e o diagnóstico pré-natal poderá ter implicações sobre a morbilidade e a mortalidade. O objetivo deste trabalho foi estudar a influência do diagnóstico pré-natal e da distância do local de parto a um centro de referência, na morbilidade imediata e mortalidade precoce de um grupo de doentes com cardiopatias congénitas complexas.

Material e Métodos: Análise retrospetiva dos doentes com cardiopatias congénitas complexas, seguidos no nosso hospital, nascidos entre 2007 e 2012.

Resultados: Identificaram-se 126 doentes com cardiopatias congénitas complexas. Em 95\% a gravidez foi vigiada desde o primeiro trimestre existindo diagnóstico pré-natal em $42 \%$. Houve relação estatisticamente significativa entre o local do parto e a existência de diagnóstico pré-natal. A cardiopatia congénita complexa mais frequente foi a transposição das grandes artérias (45,2\%), seguida da atresia da pulmonar com comunicação interventricular $(17,5 \%)$ e ventrículo esquerdo hipoplásico $(9,5 \%)$. Oitenta e dois doentes $(65,1 \%)$ foram medicados com prostaglandinas e 38 (30,2\%) foram ventilados antes de uma intervenção. A cirurgia ocorreu no período neonatal em $73 \%$. A sobrevida atuarial aos 30 dias, 12 e 24 meses foi $85 \%$, 80\% e 75\%, respetivamente. Não houve relação estatisticamente significativa entre diagnóstico pré-natal e mortalidade.

Discussão: A maioria dos doentes com cardiopatias congénitas complexas não teve diagnóstico pré-natal. Nos casos com diagnóstico pré-natal houve referenciação e parto num centro terciário. Não houve associação estatisticamente significativa entre diagnóstico prénatal e mortalidade neonatal, como já descrito em séries heterogéneas de cardiopatia congénita complexa.

Conclusão: A maioria dos doentes com cardiopatias congénitas complexas não teve diagnóstico pré-natal. Estes dados devem ser tomados em conta no planeamento do diagnóstico pré-natal das cardiopatias congénitas.

Palavras-chave: Cardiopatias Congénitas; Diagnóstico Pré-natal.

\section{ABSTRACT}

Introduction: Complex congenital heart disease is a group of severe conditions. Prenatal diagnosis has implications on morbidity and mortality for most severe conditions. The purpose of this work was to evaluate the influence of prenatal diagnosis and distance of residence and birth place to a reference center, on immediate morbidity and early mortality of complex congenital heart disease.

Material and Methods: Retrospective study of complex congenital heart disease patients of our Hospital, born between 2007 and 2012.

Results: There were 126 patients born with complex congenital heart disease. In 95\%, pregnancy was followed since the first trimester, with prenatal diagnosis in $42 \%$. There was a statistically significant relation between birth place and prenatal diagnosis. Transposition of great arteries was the most frequent complex congenital heart disease $(45.2 \%)$, followed by pulmonary atresia with ventricular septal defect (17.5\%) and hypoplastic left ventricle (9.5\%). Eighty-two patients (65.1\%) had prostaglandin infusion and 38 (30.2\%) were ventilated before an intervention. Surgery took place in the neonatal period in $73 \%$. Actuarial survival rate at 30 days, 12 and 24 months was $85 \%, 80 \%$ and $75 \%$, respectively. There was no statistically significant relation between prenatal diagnosis and mortality. Discussion: Most patients with complex congenital heart disease did not have prenatal diagnosis. All cases with prenatal diagnosis were born in a tertiary center. Prenatal diagnosis did not influence significantly neonatal mortality, as already described in other studies with heterogeneous complex heart disease.

Conclusion: prenatal diagnosis of complex congenital heart disease allowed an adequate referral. Most patients with complex congenital heart disease weren't diagnosed prenatally. This data should be considered when planning prenatal diagnosis of congenital heart disease.

Keywords: Heart Defects, Congenital; Prenatal Diagnosis.

\section{INTRODUÇÃO}

As cardiopatias congénitas são as malformações congénitas major mais comuns e constituem o grupo com maior relevância clínica, com uma prevalência de $0,5 \%$ a $1 \%$ no período neonatal. ${ }^{1-3}$ Cerca de metade destas cardiopatias são graves e necessitam de intervenção no período neonatal ou nos primeiros anos de vida. ${ }^{2}$
As cardiopatias congénitas complexas (CCC) constituem um grupo de doenças graves e incluem na sua grande maioria uma alteração do arranjo segmentar, sendo exemplos o síndrome de coração esquerdo hipoplásico (SCEH), transposição das grandes artérias (TGA) e o coração univentricular. De entre as cardiopatias congénitas,

1. Serviço de Pediatria. Hospital Vila Franca de Xira. Vila Franca de Xira. Portugal.

2. Serviço de Pediatria. Hospital de Caldas da Rainha. Centro Hospitalar Oeste. Caldas da Rainha. Portugal.

3. Serviço de Cardiologia Pediátrica. Hospital de Santa Cruz. Centro Hospitalar de Lisboa Ocidental. Lisboa. Portugal

Recebido: 21 de Agosto de 2014 - Aceite: 20 de Janeiro de 2015 | Copyright @ Ordem dos Médicos 2015 
estas são as mais graves e de mais difícil abordagem, pelo que constituem um desafio do diagnóstico à terapêutica. Alguns estudos têm demonstrado que em algumas destas cardiopatias (como a TGA), o diagnóstico pré-natal (DPN), por permitir uma programação adequada e o parto numa maternidade com articulação com centros de referência de Cardiologia e Cirurgia Cardíaca Pediátricas, tem implicações positivas sobre a morbilidade e a mortalidade..$^{4-7}$

Em Portugal não existem, até à data, estudos publicados acerca do benefício do DPN neste grupo de patologias. A cobertura nacional de ecocardiografia fetal e portanto de DPN de cardiopatias congénitas, é aparentemente assimétrica, estando disponível essencialmente nos grandes centros.

\section{OBJETIVOS}

Estudar a influência do DPN e distância do local de parto a um centro de referência com ecocardiografia fetal, na morbilidade imediata e taxa de mortalidade de um grupo de doentes com CCC, definidas como alteração do arranjo segmentar, incluindo síndrome coração esquerdo hipoplásico, transposição de grandes vasos, coração univentricular e atrésias valvulares).

\section{MATERIAL E MÉTODOS}

Estudo casuístico das crianças com CCC (definida como qualquer alteração no arranjo segmentar cardíaco) seguidas no Serviço de Cardiologia Pediátrica do nosso hospital e nascidas entre Janeiro de 2007 e Dezembro de 2012. Os doentes com diagnóstico pré-natal de CCC e com morte fetal in útero ou em que houve interrupção de gravidez foram excluídos do estudo. Procedeu-se à análise dos processos clínicos para determinação dos seguintes parâmetros: data de nascimento, género, conselho de residência, diagnóstico, vigilância de gravidez, existência ou não de diagnóstico pré-natal (DPN), idade gestacional à data do diagnóstico de CCC, local do parto, terapêutica com prostaglandinas, necessidade de ventilação e acidose grave $(\mathrm{pH}$ $<7,2$ ) previamente à primeira intervenção, tipo e data da primeira intervenção realizada (cateterismo ou cirurgia) e mortalidade. Relativamente ao concelho de residência e ao local do parto, procedeu-se à análise da distância a um centro terciário com cardiologia pediátrica, sendo englobados nos seguintes grupos: 0 (quando o conselho de resi- dência/local do parto era o mesmo de centros terciários), < 100 km, 101 a 200 km, 201 a 300 km, > 300 km, ilhas e no estrangeiro, em países sem DPN. Compararam-se grupos com e sem DPN e grupos com parto a diferentes distâncias do centro terciário. A análise estatística foi realizada através do PASW Statistics $18^{\circledR}(p<0,05)$.

\section{RESULTADOS}

Nos 6 anos abrangidos pelo estudo, foram diagnosticados 126 recém-nascidos com CCC de acordo com a definição anterior. A distribuição anual dos nascimentos está representada na Fig. 1. A distância da residência a um centro terciário de cardiologia pediátrica está indicada na Tabela 1 , não se tendo verificado diferença estatisticamente significativa entre os grupos com e sem DPN. A relação género masculino/feminino foi de 1,8:1.

A gravidez foi vigiada em 124 casos (98,4\%) e em 120 a partir do primeiro trimestre. A idade do diagnóstico está representada na Tabela 1. Houve DPN em 42 doentes com CCC. O local do parto foi no mesmo concelho de um centro terciário de cardiologia pediátrica em 64 (Tabela 1), havendo uma diferença estatisticamente significativa entre o local do parto e a existência de DPN $(p<0,001)$. Todas as crianças com DPN que residiam a mais de $100 \mathrm{~km}$ de um centro terciário tiveram o parto programado para ocorrer nesse centro e não na área da residência.

A CCC mais frequente foi a transposição das grandes artérias $(45,2 \%)$, seguida da atrésia da pulmonar com comunicação interventricular (17,5\%) (Fig. 2), havendo um número mais elevado de DPN nas CCC mais graves, como a síndrome de coração esquerdo hipoplásico (SCEH).

Foram medicados com prostaglandinas 82 doentes $(65,1 \%), 31$ dos quais com DPN. Estiveram ventilados antes da intervenção cirúrgica 38 doentes $(30,2 \%)$, dos quais 17 correspondendo a situações em que a ventilação não está habitualmente indicada e em que pode ser deletéria ou inapropriada. Nove doentes apresentaram acidose grave pré-intervenção, não havendo relação estatisticamente significativa $(p=0,06)$ entre a ausência de DPN e estas ocorrências de necessidade de ventilação ou acidose grave. Analisando a acidose e ventilação em conjunto, não houve diferença estatisticamente significativa no outcome dos grupos com e sem DPN ( $p=0,892)$. A regressão logística para o outcome composto tendo em conta DPN e local

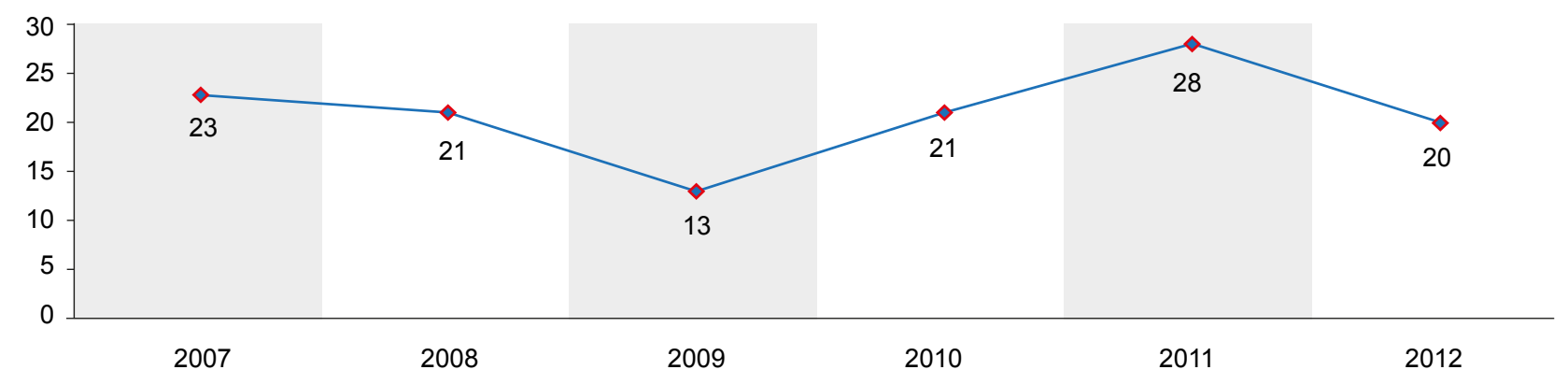

Figura 1 - Distribuição anual nascimento doentes com CCC 
Tabela 1 - Características das CCC com e sem DPN referenciadas ao nosso hospital, entre 2007 e 2012

\begin{tabular}{|c|c|c|c|}
\hline Variável & Com DPN (\%) & Sem DPN (\%) & Valor $p$ \\
\hline $\operatorname{CCC}$ & $42(33)$ & $84(67)$ & \\
\hline Distância residência a centro terciário & & & 0,09 \\
\hline $0 \mathrm{~km}$ & 10 & 8 & \\
\hline$\leq 100 \mathrm{~km}$ & 12 & 44 & \\
\hline $101-200 \mathrm{~km}$ & 3 & 2 & \\
\hline $201-300 \mathrm{~km}$ & 10 & 14 & \\
\hline$>300 \mathrm{~km}$ & 2 & 3 & \\
\hline Regiões autónomas & 5 & 12 & \\
\hline Não identificado & 0 & 1 & \\
\hline \multicolumn{4}{|l|}{ Idade diagnóstico } \\
\hline$<24$ SG & $20(16)$ & NA & \\
\hline$\geq 24$ SG & $22(17)$ & NA & \\
\hline $1^{\mathrm{a}}$ semana de vida & NA & $70(56)$ & \\
\hline $2^{\mathrm{a}}$ semana de vida & NA & $5(4)$ & \\
\hline$>2^{\mathrm{a}}$ semana de vida & NA & $9(7)$ & \\
\hline Distância local parto a centro terciário & & & 0,001 \\
\hline $0 \mathrm{~km}$ & 42 & 23 & \\
\hline$\leq 100 \mathrm{~km}$ & 0 & 27 & \\
\hline $101-200$ km & 0 & 0 & \\
\hline $201-300 \mathrm{~km}$ & 0 & 15 & \\
\hline$>300 \mathrm{~km}$ & 0 & 1 & \\
\hline Regiões autónomas & 0 & 14 & \\
\hline Não identificado & 0 & 4 & \\
\hline
\end{tabular}

NA - não se aplica

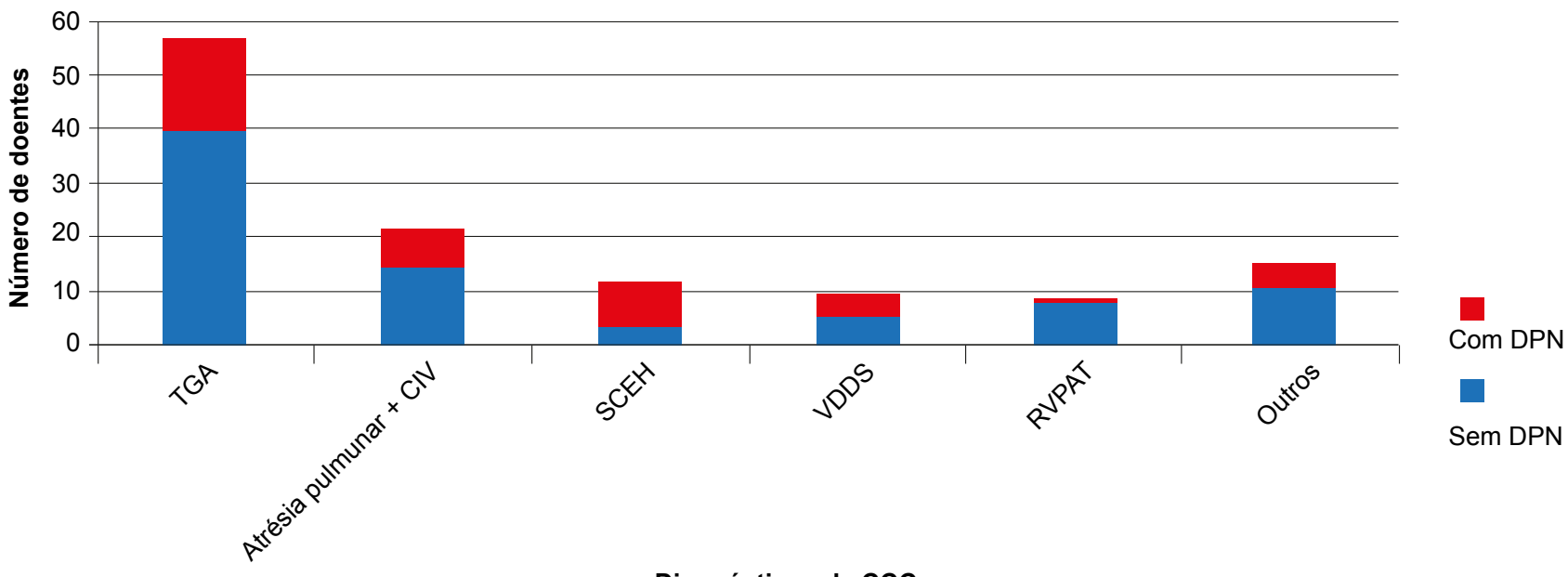

Diagnósticos de CCC

Figura 2 - Doentes com CCC referenciados ao nosso hospital no período neonatal, entre 2007 e 2012

TGA = Transposição Grandes Artérias; CIV = Comunicação interventricular; SCEH = Síndrome do Coração Esquerdo Hipoplásico; VDDS = Ventrículo Direito de Dupla Saída; RVPAT = Retorno Venoso Pulmonar Anómalo Total.

de parto também não teve diferenças.

A cirurgia ocorreu no período neonatal em $73 \%$ dos doentes (sendo esta a primeira intervenção em 60\%). A mediana da idade das crianças na primeira intervenção foi de 7 dias. Em 76 doentes procedeu-se a uma cirurgia e realizou-se cateterismo terapêutico em 46 (Tabela 2). Nos casos em que houve cateterismo terapêutico, este foi na maioria dos casos a septostomia de Rashkind (41). Nas TGA, a septostomia foi realizada em média ao segundo dia de vida. A cirurgia foi correctiva em 70 crianças $(55,6 \%)$. 0 
Tabela 2 - Intervenções realizadas nas CCC referenciadas ao nosso hospital: 2007 a 2012

\begin{tabular}{|c|c|c|}
\hline Variável & $\mathbf{n}$ & $\%$ \\
\hline \multicolumn{3}{|l|}{$1^{\mathrm{a}}$ intervenção } \\
\hline Cateterismo & 46 & 36,5 \\
\hline Cirurgia & 76 & 60,3 \\
\hline Cateterismo+Cirurgia & 2 & 1,6 \\
\hline Não realizada & 2 & 1,6 \\
\hline \multicolumn{3}{|l|}{ Cateterismo terapêutico } \\
\hline Septostomia de Rashkind & 41 & 32,5 \\
\hline Abordagem híbrida neonatal & 4 & 3,2 \\
\hline Valvuloplastia percutânea & 1 & 0,8 \\
\hline Não realizado & 80 & 63,5 \\
\hline \multicolumn{3}{|l|}{ Cirurgia } \\
\hline Switch arterial & 61 & 48,4 \\
\hline Shunt Blalock-Taussig & 28 & 22,2 \\
\hline Banding Artéria Pulmonar & 11 & 8,7 \\
\hline Correcção RVPAT & 6 & 4,8 \\
\hline Outros & 18 & 14,0 \\
\hline
\end{tabular}

RVPAT = Retorno Venoso Pulmonar Anómalo Total

tipo de cirurgia mais frequente foi o switch arterial (61), seguido do shunt Blalock-Taussig (28) e do banding da artéria pulmonar (11).

Verificaram-se 12 óbitos no período pós-operatório. Não houve relação estatisticamente significativa entre mortalidade no período neonatal e DPN ou distância do local do parto a um centro de referência.

A sobrevida atuarial aos 30 dias foi de $85 \%$, aos 12 meses de $80 \%$ e aos 24 meses de $75 \%$ (Fig. 3). Não houve diferença estatisticamente significativa nas curvas de sobrevida dos doentes com e sem DPN (Fig. 4).

\section{DISCUSSÃO}

Sendo as CCC o grupo de cardiopatias mais grave,

Sobrevida Global

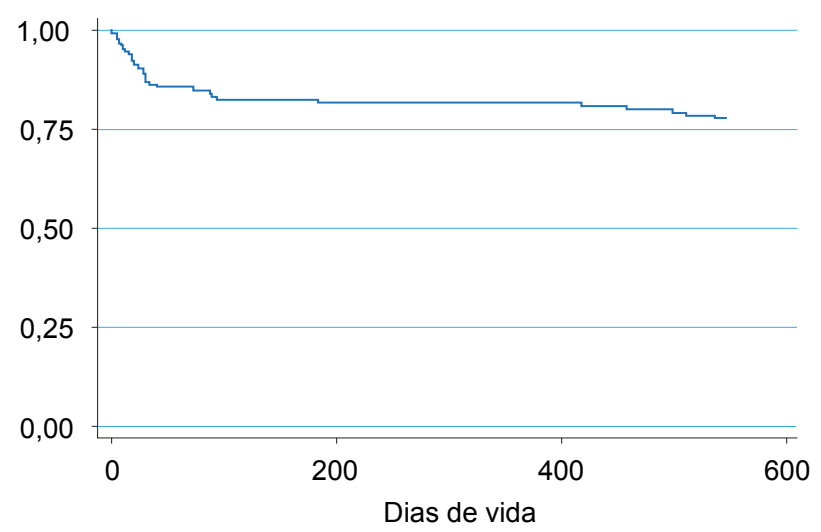

Figura 3 - Curva de sobrevida atuarial para todas as CCC salienta-se que a maioria dos doentes deste estudo não teve DPN. Na literatura, estão descritas taxas de DPN de cardiopatias congénitas de 47,3\% em França, 49\% nos Estados Unidos e $52,8 \%$ na Austrália. ${ }^{7,8,13}$ Em Portugal, a Direção Geral da Saúde publicou em 2006 um conjunto de indicações para a realização de ecocardiografia fetal, salientando que na generalidade das gravidezes, a realização de uma ecografia do segundo trimestre de qualidade permite identificar a maioria dos fetos que deverão ser submetidos a estudo ecocardiográfico. ${ }^{14} \mathrm{O}$ DPN não é tecnicamente difícil mas obriga a uma preparação adequada de todos os profissionais de saúde envolvidos neste processo, incluindo sobretudo obstetras e cardiologistas pediátricos.

Embora se esperasse que os doentes com DPN

\section{Sobrevida de acordo com DPN}

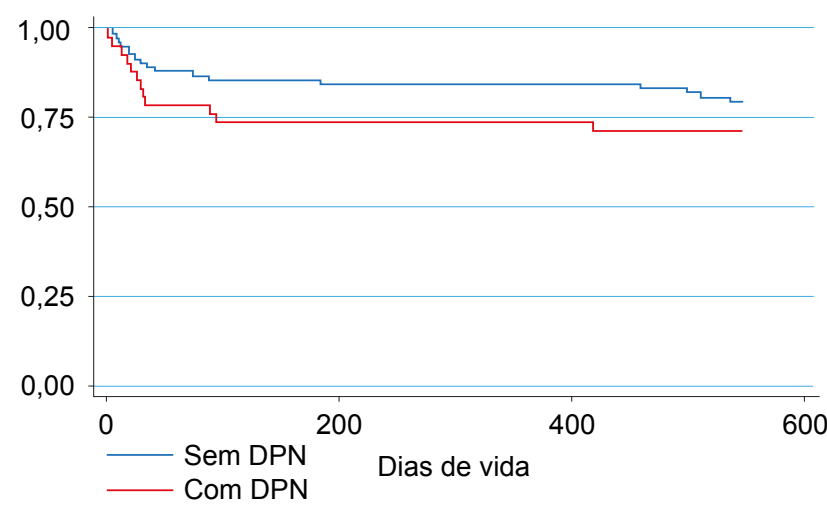

Figura 4 - Curva de sobrevida atuarial para CCC com e sem DPN. Não há diferença estatisticamente significativa entre as 2 curvas $p=0,167$. 
tivessem menor mortalidade e morbilidade, tal não se verificou. A vantagem do DPN de cardiopatias graves foi demonstrada pela primeira vez em 1999, num trabalho francês com um grupo muito homogéneo de doentes, com TGA simples. ${ }^{7}$ Noutros estudos, tem sido difícil comprovar esse benefício. ${ }^{10,11}$ Uma possível explicação para esse facto poderá resultar da heterogeneidade das $\mathrm{CCC}$ e das diferentes taxas de sobrevivência para cada patologia. Restringindo-se a amostra a determinadas cardiopatias congénitas, observa-se uma melhoria no prognóstico a curto e longo prazo quando há DPN. ${ }^{12} \mathrm{Na}$ amostra do nosso trabalho, analisando apenas as TGA, verifica-se que no grupo com DPN não existiu acidose grave, ao contrário do grupo sem DPN, onde esta ocorreu em $13 \%$. O mesmo se verifica com a ventilação, com os grupos com e sem DPN com diferenças de $24 \%$ para $43 \%$, respetivamente. No entanto, esta associação não foi estatisticamente significativa. Outro aspeto que poderá justificar a ausência aparente de benefício do DPN neste estudo é a gravidade das patologias, já que os casos mais graves serão aqueles com maior probabilidade de serem diagnosticados no período pré-natal, e serão igualmente aqueles com maior probabilidade de pior prognóstico.

Apesar do nosso estudo não demonstrar uma maior sobrevida associada ao DPN, existem seguramente vários benefícios importantes. Tem-se demonstrado que o DPN das cardiopatias complexas está associado a melhor prognóstico em várias patologias específicas, nomeadamente TGA (já que em cerca de $12 \%$ é necessário a realização de septostomia de Rashkind na primeira hora de vida, ${ }^{7}$ só possível se o parto ocorrer num centro terciário) e a uma boa predição da abordagem cirúrgica em TGA complexas ${ }^{4,9} \mathrm{O}$ DPN permite um melhor aconselhamento parental e ainda um planeamento perinatal adequado, nomeadamente no que diz respeito ao local do parto. Desta forma, o DPN leva a menores taxas de transporte pós-natal e nos casos em que este é necessário, um transporte mais curto e seguro dos recém-nascidos, designadamente com menor recurso a ventilação invasiva e a menores taxas de uso de inotrópicos..$^{15} \mathrm{Um}$ dado positivo que se destaca, é que todos os RN referenciados ao nosso centro que tiveram DPN nasceram num centro terciário, de onde se conclui que a rede de referenciação funciona quando o DPN é realizado.

Uma limitação deste estudo é o facto de se tratar de um número pequeno de doentes, referenciados a um único centro. Um outro aspecto é a ausência de alguns dados do pós-parto imediato relativos à gravidade da situação, já que muitos nascimentos ocorreram em outras unidades hospitalares.

\section{CONCLUSÕES}

Este estudo, realizado com doentes nascidos entre 2007 e 2012, mostra que a maioria dos doentes com cardiopatia congénita complexa não teve DPN, o que sublinha a necessidade de investir em todo o circuito de diagnóstico pré-natal destas patologias, desde a formação dos profissionais que realizam ecografia obstétrica, às indicações para a referenciação e estabelecimento de circuitos de referenciação após a suspeita de patologia fetal. Um ponto interessante é que para este grupo de doentes, nascidos com cardiopatia complexa, as regiões mais afastadas dos grandes centros não têm uma taxa de diagnóstico pré-natal inferior ao das regiões mais próximas dos centros de referência. De salientar que a rede de referenciação com o nosso centro funcionou em todos os casos em que o DPN foi realizado, com o parto a ocorrer em centros terciários possibilitando menor morbilidade neonatal precoce.

Este estudo é um contributo para uma reflexão mais ampla sobre a metodologia a adotar para implementar de forma mais eficaz o DPN das cardiopatias congénitas no nosso País.

\section{OBSERVAÇÕES}

Trabalho parcialmente apresentado na forma de comunicação oral, sob o título "Cardiopatias congénitas complexas e diagnóstico pré-natal: casuística de um centro de referência", nas XLII Jornadas Nacionais de Neonatologia/V Jornadas Internacionais de Neonatologia; Lisboa, 14 e 15 de Novembro de 2013.

\section{CONFLITOS DE INTERESSE}

Os autores declaram não ter qualquer conflito de interesse relativamente ao presente artigo.

\section{FONTES DE FINANCIAMENTO}

Os autores declaram não ter recebido qualquer subsídio relativo ao presente artigo.

the prevalence of liveborn congenital heart disease. Pediatr Cardiol 2006;27:465-72.

6. McBrien A, Andrew S, Craig B, Dornan J, Casey F. Major congenital heart disease: antenatal detection, patient characteristics and outcomes. J Matern Fetal Neonatal Med. 2009;22:101-5.

7. Bonnet D, Coltri A, Butera G, Fermont L, Bidois J, Kachaner J, et al. Detection of transposition of the great arteries in fetuses reduces neonatal morbidity and mortality. Circulation. 1999;99:916-8.

8. Chew C, Halliday JL, Riley MM, Penny DJ. Population-based study of antenatal detection of congenital heart disease by ultrasound examination. Ultrasound Obstet Gynecol. 2007; 29:619-24.

9. Pascal CJ, Huggon I, Sharland GK, Simpson JM. An echocardiographic study of diagnostic accuracy, prediction of surgical approach, and outcome for fetuses diagnosed with discordant ventriculo-arterial 
connections. Cardiol Young. 2007; 17:528-34

10. Mahle WT, Clancy RR, McGaurn SP, Goin JE, Clark BJ. Impact of prenatal diagnosis on survival and early neurologic morbidity in neonates with the hypoplastic left heart syndrome. Pediatrics. 2001;107:1277-82.

11. Raboisson MJ, Samson C, Ducreux C, Rudigoz RC, Gaucherand P, Bouvagnet $P$, et al. Impact of prenatal diagnosis of transposition of the great arteries on obstetric and early post natal management. Eur J Obstet Gynecol Reprod Biol. 2009;142:18-22.

12. Fuchs IB, Muller $\mathrm{H}$, Abdul-Khaliq $\mathrm{H}$, Harder $\mathrm{T}$, Dudenhausen JW, Henrich W. Immediate and long-term outcomes in children with prenatal diagnosis of selected isolated congenital heart defects. Ultrasound Obst Gynecol. 2007;29:38-43.

13. Israel SW, Roofe LR, Saville BR, Walsh WF. Improvement in antenatal diagnosis of critical congenital heart disease - implications for postnatal care and screening. Fetal Diagn Ther. 2011;30:180-3.

14. Diagnóstico Prenatal de Cardiopatias Congénitas. Circular Normativa ${ }^{\circ}$ 11/DSMIA da Direcção Geral da Saúde. Lisboa: DGS; 2006.

15. Gupta N, Leven L, Stewart M, Cheung M, Patel N. Transport of infants with congenital heart disease: benefits of antenatal diagnosis. Eur $\mathrm{J}$ Pediatr. 2014;173:655-60. 
Marta CORREIA, Fabiana FORTUNATO, Duarte MARTINS, Ana TEIXEIRA, Graça NOGUEIRA, Isabel MENEZES, Rui ANJOS

\section{Cardiopatias Congénitas Complexas: Influência do Diagnóstico Pré-Natal}

Acta Med Port 2015:28:158-163

Publicado pela Acta Médica Portuguesa, a Revista Científica da Ordem dos Médicos

Av. Almirante Gago Coutinho, 151

1749-084 Lisboa, Portugal.

Tel: +351218428 215

E-mail: submissao@actamedicaportuguesa.com

www.actamedicaportuguesa.com

ISSN:0870-399X | e-ISSN: 1646-0758

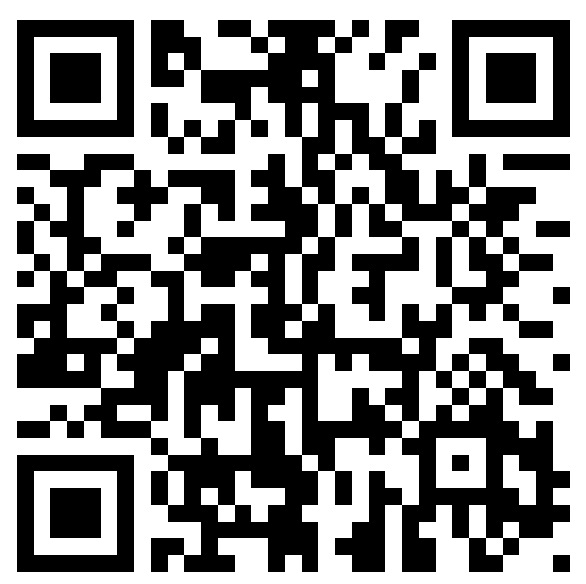

ACTA MÉDICA

PORTUGUESA 